\title{
Order from disorder in the sarcomere: FATZ forms a fuzzy complex and phase-separated condensates with $\alpha$-actinin
}

\author{
Antonio Sponga ${ }^{1,{ }^{*}}$, Joan L. Arolas ${ }^{1,{ }^{*}}$, Thomas C. Schwarz ${ }^{1}$, Cy M. Jeffries ${ }^{2}$, Ariadna Rodriguez Chamorro ${ }^{1}$, Julius \\ Kostan $^{1}$, Andrea Ghisleni ${ }^{3}$, Friedel Drepper ${ }^{4,5}$, Anton Polyansky ${ }^{1,6}$, Euripedes De Almeida Ribeiro ${ }^{1}$, Miriam \\ Pedron $^{1}$, Anna Zawadzka-Kazimierczuk ${ }^{7}$, Georg Mlynek ${ }^{1}$, Thomas Peterbauer ${ }^{8}$, Pierantonio Doto ${ }^{1}$, Claudia \\ Schreiner ${ }^{1}$, Eneda Hollerl ${ }^{1}$, Borja Mateos ${ }^{1}$, Leonhard Geist ${ }^{1}$, Georgine Faulkner ${ }^{9}$, Wiktor Kozminski ${ }^{7}$, Dmitri I. \\ Svergun $^{3}$, Bettina Warscheid ${ }^{4,5}$, Bojan Zagrovic ${ }^{1}$, Mathias Gautel ${ }^{3}$, Robert Konrat ${ }^{1}$, Kristina Djinović-Carugo $^{1,10, \$}$ \\ ${ }^{1}$ Department of Structural and Computational Biology, Max Perutz Labs, University of Vienna, Campus Vienna Biocenter 5, A-1030 \\ Vienna, Austria \\ ${ }^{2}$ European Molecular Biology Laboratory (EMBL), Hamburg Unit, Hamburg, Germany \\ ${ }^{3}$ King's College London BHF Centre for Research Excellence, Randall Centre for Cell and Molecular Biophysics, \\ London SE1 1UL, UK \\ ${ }^{4}$ Biochemistry and Functional Proteomics, Institute of Biology II, Faculty of Biology, University of Freiburg, \\ 79104 Freiburg, Germany \\ ${ }^{5}$ Signalling Research Centres BIOSS and CIBSS, University of Freiburg, 79104 Freiburg, Germany \\ ${ }^{6}$ National Research University Higher School of Economics, Moscow 101000, Russia \\ ${ }^{7}$ Biological and Chemical Research Centre, Faculty of Chemistry, University of Warsaw, Zwirki i Wigury 101, \\ 02-089 Warsaw, Poland \\ ${ }^{8}$ Department of Biochemistry and Cell Biology, Max Perutz Labs, University of Vienna, Dr. BohrGasse 9, A-1030 Vienna, Austria \\ ${ }^{9}$ Department of Biology, University of Padova, 35100 Padova, Italy \\ ${ }^{10}$ Department of Biochemistry, Faculty of Chemistry and Chemical Technology, University of Ljubljana, Večna pot 113, SI-1000 \\ Ljubljana, Slovenia \\ kristina.djinovic@univie.ac.at
}

In sarcomeres, $\alpha$-actinin crosslinks actin filaments and anchors them to the Z-disk. FATZ proteins interact with $\alpha$-actinin and five other core Z-disk proteins, contributing to myofibril assembly and maintenance as a protein interaction hub.

Here we report the first structure and its cellular validation of $\alpha$-actinin- 2 in complex with a Z-disk partner, FATZ-1, which is best described as a conformational ensemble. We show that FATZ-1 forms a tight fuzzy complex with $\alpha$-actinin- 2 and propose a molecular interaction mechanism via main molecular recognition elements and secondary binding sites. The obtained integrative model reveals a polar architecture of the complex which, in combination with FATZ-1 multivalent scaffold function, might organise interaction partners and stabilise $\alpha$-actinin-2 preferential orientation in the Z-disk.

Finally, we uncover FATZ-1 ability to phase-separate and form biomolecular condensates with $\alpha$-actinin-2, raising the intriguing question whether FATZ proteins can create an interaction hub for Z-disk proteins through membrane-less compartmentalization during myofibrillogenesis.

Keywords: striated muscle Z-disk; $\alpha$-actinin; FATZ; protein-protein interaction hub; intrinsic disorder; tight fuzzy complex; liquid-liquid phase separation; integrative structural biology; 